\title{
SARS-CoV-2 antibodies in the Southern Region of New Zealand, 2020
}

\author{
Alyson Craigie ${ }^{1,2}$, Reuben McGregor ${ }^{3,4}$, Alana L. Whitcombe ${ }^{3,4}$, \\ Lauren Carlton ${ }^{3,4}$, David Harte ${ }^{5}$, Michelle Sutherland ${ }^{5}$, \\ Matthew Parry ${ }^{6}$, Erasmus Smit ${ }^{5}$, Gary McAuliffe ${ }^{7,8}$, James Ussher ${ }^{1,2}$, \\ Nicole J. Moreland ${ }^{3,4}$, Susan JaCK ${ }^{9}$, Arlo Upton ${ }^{1}$ \\ ${ }^{1}$ Southern Community Laboratories, Dunedin, New Zealand; ${ }^{2}$ Department of Microbiology \\ and Immunology, University of Otago, Dunedin, New Zealand; ${ }^{3}$ Faculty of Medical and \\ Health Sciences, University of Auckland, Auckland, New Zealand; ${ }^{4}$ Maurice Wilkins Centre, \\ University of Auckland, Auckland, New Zealand; ${ }^{5}$ Institute of Environmental Science and \\ Research, Wellington, New Zealand; ${ }^{6}$ Department of Mathematics and Statistics, University \\ of Otago, Dunedin, New Zealand; ${ }^{7}$ Labtests, Auckland, New Zealand; ${ }^{8}$ LabPlus, Auckland \\ Hospital, Auckland, New Zealand; ${ }^{9}$ Public Health South, Southern District Health Board, \\ Dunedin, New Zealand
}

\begin{abstract}
Summary
During New Zealand's first outbreak in early 2020 the Southern Region had the highest per capita SARS-CoV-2 infection rate. Polymerase chain reaction (PCR) testing was initially limited by a narrow case definition and limited laboratory capacity, and cases may have been missed. Our objectives were to evaluate the Abbott SARS-CoV-2 $\operatorname{IgG}$ nucleocapsid assay, alongside spike-based assays, and to determine the frequency of antibodies among PCRconfirmed and probable cases, and higher risk individuals in the Southern Region of New Zealand.

Pre-pandemic sera $(n=300)$ were used to establish assay specificity and sera from PCR-confirmed SARS-CoV-2 patients $(n=78)$ to establish sensitivity. For prevalence analysis, all samples $(n=1214)$ were tested on the Abbott assay, and all PCR-confirmed cases $(n=78)$, probable cases $(n=9)$, and higher risk individuals with 'grey-zone' ( $n=14)$ or positive results $(n=11)$ were tested on four additional SARS-CoV-2 serological assays.

The median time from infection onset to serum collection for PCR-confirmed cases was 14 weeks (range 11-17 weeks). The Abbott assay demonstrated a specificity of $99.7 \%(95 \% \mathrm{Cl} 98.2-99.99 \%)$ and a sensitivity of $76.9 \%$ (95\% Cl 66.0-85.7\%). Spike-based assays demonstrated superior sensitivity ranging $89.7-94.9 \%$. Nine previously undiagnosed sero-positive individuals were identified, and all had epidemiological risk factors.

Spike-based assays demonstrated higher sensitivity than the Abbott IgG assay, likely due to temporal differences in antibody persistence. No unexpected SARS-CoV-2 infections were found in the Southern Region of New Zealand, supporting the elimination status of the country at the time this study was conducted.
\end{abstract}

Key words: SARS-CoV-2; COVID-19; antibodies; nucleocapsid; spike.

Received 27 October 2020, revised 5 April, accepted 7 April 2021 Available online 18 May 2021

\section{INTRODUCTION}

The novel virus Severe Acute Respiratory Syndrome Coronavirus 2 (SARS-CoV-2) and the disease it causes, COVID19, were first detected in Wuhan, China in December 2019. The World Health Organization (WHO) declared COVID-19 a public health emergency of international concern on 30 January 2020, and a pandemic on 11 March $2020 .^{2}$ As of 8 February 2021, there have been over 106 million confirmed COVID-19 cases worldwide, with over 2.3 million deaths. ${ }^{3}$ During New Zealand's (NZ) first outbreak of COVID-19 (28 February to 22 May 2020) a total of 1154 polymerase chain reaction (PCR)-confirmed and 350 'probable' (symptomatic household contacts of PCR-positive cases who had negative PCR testing) cases were identified with 22 COVID19 related deaths. ${ }^{4}$

NZ initially responded to the pandemic in early February 2020 by stopping foreign nationals from affected countries entering NZ and enforcing self-isolation for NZ citizens and permanent residents travelling from these countries. ${ }^{5}$ On 21 March 2020, a four-tier alert system was introduced; the country started in Level 2, and quickly moved to the highest alert Level 4 restrictions on 26 March. ${ }^{5}$ Alert Level 4 is a national lockdown with a strict stay at home order for all but essential workers. ${ }^{6} \mathrm{NZ}$ remained at Alert Level 4 for approximately 5 weeks before a stepped de-escalation to Alert Level 1 on 8 June $2020 .^{5}$

This serological study is focused on the Southern District Health Board (SDHB) region in NZ which had the largest number of cases per capita during the first outbreak of COVID-19 (216 total cases; $\sim 66 / 100,000$ population), significantly higher than the national average $(\sim 30 / 100,000)$. This region also includes the tourism hub of Queenstown, where community transmission took place. PCR testing for SARS-CoV-2 was initially restricted during the first outbreak in NZ due to a narrow case definition and limited access to diagnostic reagents.

Reverse transcription-PCR (RT-PCR, henceforth referred to as PCR) from a nasopharyngeal and/or oropharyngeal swab 
or lower respiratory tract sample is the gold standard method for detecting acute SARS-CoV-2 infection whereas serological tests can provide information on past infection, including where patients have been symptomatic for some time and are PCR negative. ${ }^{7}$ SARS-CoV-2 has four structural proteins: Spike (S), Membrane (M), Envelope (E), and Nucleocapsid $(\mathrm{N})$, with the majority of the serological assays developed to detect antibodies against the $\mathrm{S}$ and/or $\mathrm{N}$ protein. ${ }^{7}$ Differing degrees of protein sequence conservation between the $\mathrm{N}$ and $\mathrm{S}$ proteins [including S1 and receptor binding domains (RBD)], and proteins from other coronavirus species, together with differences in the magnitude and kinetics of the antibody response to these antigens may impact assay performance. Several serological assays are now commercially available, including for use on high-throughput, random access analysers such as the Abbott Architect.

The aims of this study were threefold. Firstly, to investigate the sensitivity and specificity of the Abbott Architect SARS-CoV-2 IgG assay based on the N-protein, together with a series of plate-based assays that utilise the $S$ protein and/or $S$ protein domains, including a surrogate viral neutralisation assay. Secondly, to determine the frequency of SARS-CoV-2 IgG antibodies among higher risk individuals in the Southern Region of NZ to determine whether cases were missed during the outbreak due to limited PCR testing and/or asymptomatic infection. The third aim was to use serological testing to assess the likelihood of infection among those diagnosed as 'probable' cases.

\section{MATERIALS AND METHODS}

\section{Study protocol}

This study was performed at Southern Community Laboratories, Dunedin, NZ, in conjunction with the Southern District Health Board (SDHB), WellSouth (the local primary healthcare organisation), University of Otago, University of Auckland, and the Institute of Environmental Science and Research (ESR). Ethical approval for this project was obtained from the NZ Health and Disability Ethics (HDEC) Committee (20/NTB/101).

\section{Patient cohorts}

In total 1214 individuals gave informed consent and participated in the study, with bloods collected between 4 June and 4 August 2020, 4-10 weeks after active transmission in the community in the Southern Region ceased. Of these, 78 were PCR-confirmed cases, nine were probable cases, and 1127 individuals were in the higher risk group.

\section{Case definitions}

Confirmed and probable cases were classified according to the NZ Ministry of Health $(\mathrm{MoH})$ guidelines. Confirmed cases were positive by PCR; probable cases were PCR negative, a household contact of a confirmed case, and had a clinically compatible illness with other causes excluded. ${ }^{9}$ COVID-19 consistent symptoms were defined by the $\mathrm{MoH}$ as any acute respiratory infection with at least one of the following symptoms (with or without fever): new or worsening cough, sore throat, shortness of breath, coryza, anosmia. ${ }^{10}$

The higher risk group was considered to be of higher risk than the general population of having undiagnosed COVID-19 infection, either by contact with a PCR-confirmed case or because of workplace duties (frontline healthcare workers, tourism workers), or were Queenstown residents. While some of the higher risk group may appear to fit the definition of a probable case, they were not under public health management and therefore were not categorised as probable cases; their contact and symptoms were self-identified retrospectively.

The severity of COVID-19 infection in the PCR-confirmed group were classified on the basis of their symptoms and the level of hospital care provided: 1 , asymptomatic to mild cold-like symptoms $(n=35) ; 2$, moderate: cough, fever and chills $(n=39) ; 3$, moderately severe: admitted for assessment $(n=3) ; 4$, severe: admitted and given supplemental oxygen therapy $(n=1) ; 5$, critical: admitted to ICU $(n=0)$.

To determine assay specificity, 300 de-identified antenatal sera collected from early-mid 2019 (pre-pandemic), were used (after being stored at $-20^{\circ} \mathrm{C}$ for up to 12 months as per laboratory protocols). To determine sensitivity, samples from the 78 PCR-confirmed cases were used.

The PCR-confirmed and probable cases, and their contacts were contacted for recruitment via the local public health unit. The remaining higher risk individuals were recruited via posters and media (print, television, and social media). Participants completed a Research Electronic Data Capture (REDCap) online survey. ${ }^{11}$ The questionnaire included demographic details and a set of questions, including which higher risk category they associated with, if they had any contact with known COVID-19 cases, and whether they recalled having symptoms consistent with COVID-19 before or during the lockdown period (alert Level 3 and 4, between 23 March and 12 May 2020) (Table 1).

\section{PCR assays}

Participants who had PCR testing were tested using one of four assays that were in use during the first outbreak, all performed at SCL Dunedin: (1) an inhouse real time RT-PCR assay targeting the E-gene based on the Drosten assay $^{12}$ and implemented on the open access channel of the Hologic Panther Fusion (Hologic, USA); (2) a multiplex tandem real-time RT-PCR SARSCoV-2, Influenza, and RSV (8-well) assay (AusDiagnostics, Australia); (3) TaqPath COVID-19 Combo assay (ThermoFisher Scientific, USA), a multiplex real-time RT-PCR; and (4) the Aptima SARS-CoV-2 Assay (Hologic), a transcription mediated amplification assay.

\section{Serological assays}

A summary of the assays utilised is shown in Table 2 . The primary assay was the Abbott Architect SARS-CoV-2 IgG chemiluminescent microparticle immunoassay (CMIA; Abbott, USA), which uses recombinant $\mathrm{N}$ protein coated microparticles. Samples were analysed on the Abbott Architect i2000SR Immunoassay Analyzer following manufacturer's instructions. Test performance for the Abbott assay was assessed using the manufacturer's cutoff for positivity $(\geq 1.4 \mathrm{~S} / \mathrm{C})$.

The in house two-step ELISA was adapted from published protocols ${ }^{13}$ as described. ${ }^{14}$ In step one, serum diluted 1:100 was screened against RBD, with IgG binding detected with a peroxidase-labelled anti-human IgG secondary. Samples with an optical density (OD, $450-570 \mathrm{~nm}$ ) above the cut-off $(>0.2)$ were titrated in a 3 -fold dilution series against the $S$ protein in step two and considered positive if they had an OD $>0.2$ in at least two consecutive wells in the confirmatory S protein ELISA; the result was reported as the highest titre above the cut-off $(>0.2)$.

The Wantai SARS-CoV-2 ELISA (Beijing Wantai Biological Pharmacy Enterprise, China) measures total antibody (IgA, IgG and IgM) against the $\mathrm{RBD}$ of the $\mathrm{S}$ protein. The Euroimmun SARS-CoV-2 IgG ELISA (Euroimmun Medizinische Labordiagnostika, Germany) measures IgG antibodies against the spike S1 subunit. The Wantai and the Eurommun ELISAs were performed according to the manufacturer's instructions.

The cPass surrogate viral neutralisation test (sVNT) (GenScript, New Jersey, USA) measures the presence of neutralising antibodies that are capable of blocking the interaction between RBD and hACE2 ${ }^{15}$ and was performed according to the manufacturer's instructions. Samples with a percentage inhibition $\geq 20 \%$ were defined as having neutralising antibodies.

To assess cross-reactivity of the antenatal sera $(n=300)$ with other human coronaviruses ( $\mathrm{HCoV})$, ELISA were performed using S1 antigens from the HKU1, NL63, and SARS-CoV-2 (Sino Biological, China) at a 1:300 sera dilution as described. ${ }^{11}$

\section{Testing protocol}

The 300 specificity samples and the serum from PCR-confirmed and probable cases were tested on all five assays. All sera from the higher risk group ( $n=1127$ ) were tested on the Abbott assay. Samples from the higher risk group that classified as positive $(\geq 1.4 \mathrm{~S} / \mathrm{C})$, or as negative but $\geq 0.5$ $\mathrm{S} / \mathrm{C}$ [i.e., 0.5-1.39 S/C, defined as the 'grey-zone' on the basis of a receiver operating characteristic (ROC) curve] were tested on all assays $(n=25)$. 
Table 1 Patient demographics

\begin{tabular}{|c|c|c|c|c|}
\hline & Total & PCR-confirmed cases & Probable cases & Higher risk group \\
\hline Total & $1214(100 \%)$ & $78(6 \%)$ & $9(1 \%)$ & $1127(93 \%)$ \\
\hline Median & 46 & 51 & 49 & 46 \\
\hline Range & $4-90$ & $17-81$ & $10-59$ & $4-90$ \\
\hline \multicolumn{5}{|l|}{ Gender } \\
\hline $\mathrm{M} / \mathrm{F}$ & $306 / 908$ & $32 / 46$ & $3 / 6$ & $271 / 856$ \\
\hline Frontline healthcare workers & & & & $702(62 \%)$ \\
\hline Tourism worker & & & & $60(5 \%)$ \\
\hline Queenstown resident & & & & $208(19 \%)$ \\
\hline One or more COVID-19 consistent symptoms reported & & & & $466(41 \%)$ \\
\hline
\end{tabular}

\section{Statistical analysis}

Statistical analysis, including ROC curve, was performed using Prism 8 (GraphPad, USA) or R (version 3.6.3) within R Studio (version 1.2.5033); a $p$ value of $\leq 0.05$ was considered statistically significant. To assess sensitivity, serological assays were compared against PCR diagnosed COVID-19. Equivocal results were considered negative for the statistical analyses. For the higher risk group, true sero-positivity was defined as positivity in two or more of the five assays. False positivity was defined as positivity in only one of the five assays.

\section{RESULTS}

\section{Participant characteristics}

As shown in Table 1, the median age of the mostly female (75\%) participants was 46 years (range $4-90$ years). Of the 1127 higher risk participants, 37\% had had a PCR test (all negative), $62 \%$ self-identified as frontline healthcare workers in the SDHB region, and $41 \%$ retrospectively reported one or more symptoms consistent with COVID-19 in the two weeks leading up to and during the February-May 2020 COVID-19 outbreak. For the PCR-confirmed and probable cases, the median time of symptom onset to serology specimen collection was 14 weeks (range 11-17 weeks).

\section{Assay performance}

The overall performance of the assays is summarised in Table 3. Specificity was high across all assays ranging from $99.3 \%$ [95\% confidence interval (CI) $97.6-99.9 \%$ ] to $100 \%$ (95\% CI 98.8-100.0\%) (Supplementary Tables 1-4, Appendix A). The antenatal sera used to determine specificity showed broad reactivity with $\mathrm{S} 1$ protein antigens from $\mathrm{HCoV}$ (HKU1 and NL63), but not SARS-CoV-2 (Supplementary Fig. 1, Appendix A).

Sensitivity ranged from $76.9 \%$ (95\% CI $66.0-85.7 \%)$ for the Abbott assay, to $94.9 \%$ (95\% CI $87.4-98.6 \%$ ) for the Wantai assay (Fig. 1, Table 3). Eighteen of the 78 (23.1\%) PCR-confirmed cases tested negative on the Abbott (Supplementary Table 2, Appendix A). The raw values for these ranged from $0.14-1.39 \mathrm{~S} / \mathrm{C}$. Eleven of these were positive on three or more of the other assays, four were positive on two of the other assays, one was positive on one of the other assays, and two were negative on all the other assays.

The sensitivity of the Abbott assay was unexpectedly low and prompted a ROC analysis that showed a cut-off of $0.55 \mathrm{~S} /$ $\mathrm{C}$ could achieve much greater sensitivity $(93.6 \%)$ without a significant loss in specificity (98.7\%) (Supplementary Fig. 2,
Appendix A). Therefore, a grey-zone approach was utilised for analysis of the higher risk group to rule out potential false negatives. Any samples that fell between $0.5-1.39 \mathrm{~S} / \mathrm{C}$ were measured on the other four assays.

\section{Neutralising anti-SARS-CoV-2 antibodies}

The sVNT assay was used to assess the presence of neutralising antibodies (NAbs). For the PCR-confirmed group, $88.5 \%$ (69/78) had detectable NAbs (Supplementary Table 2, Appendix A), illustrating the majority of individuals retain functional antibodies for at least 3 months post-infection. When the PCR-confirmed patients were stratified by disease severity, there was a small but significant increase in the level of NAbs in those with more severe disease $(p<0.05)$ (Supplementary Table 3, Appendix A).

\section{Antibody detection among higher risk individuals}

Eleven individuals of the higher risk group (0.98\%) had positive results on the Abbott assay (Fig. 1). Eight of these were also positive on one or more of the other four assays, indicating true sero-positivity. Three Abbott positive results were therefore considered false positives as they were negative on all four other assays. There were 14 Abbott results that fell in the grey-zone $(0.5-1.39 \mathrm{~S} / \mathrm{C})$. Thirteen $(93 \%)$ were negative on all four other assays and classified as seronegative. One individual was positive on all four other assays (with travel history and symptoms) and considered seropositive (Supplementary Fig. 3, Appendix A).

Thus, in total we detected nine additional possible COVID19 infections; one was a PCR-confirmed case diagnosed outside of the Southern Region; six had consistent travel history (Western Europe/UK) and symptoms; and two were close contacts of PCR-confirmed cases reporting consistent symptoms.

\section{Estimation of actual prevalence in the higher risk group}

We detected 9/1127 (0.8\%) sero-positive individuals in the higher risk group but to estimate the true number of cases of infection that may have been missed, we conducted the following statistical analyses to assess the reliability of this estimate. The Rogan-Gladen estimator ${ }^{16}$ allowed us to estimate the prevalence in the higher-risk group, taking into account the uncertainties in the sensitivity and specificity of the test. Using the Abbott assay with a threshold of $1.4 \mathrm{~S} / \mathrm{C}$, the estimated prevalence in the higher-risk group is $0.8 \%$ 
Table 2 Summary of the investigated SARS-CoV-2 assays

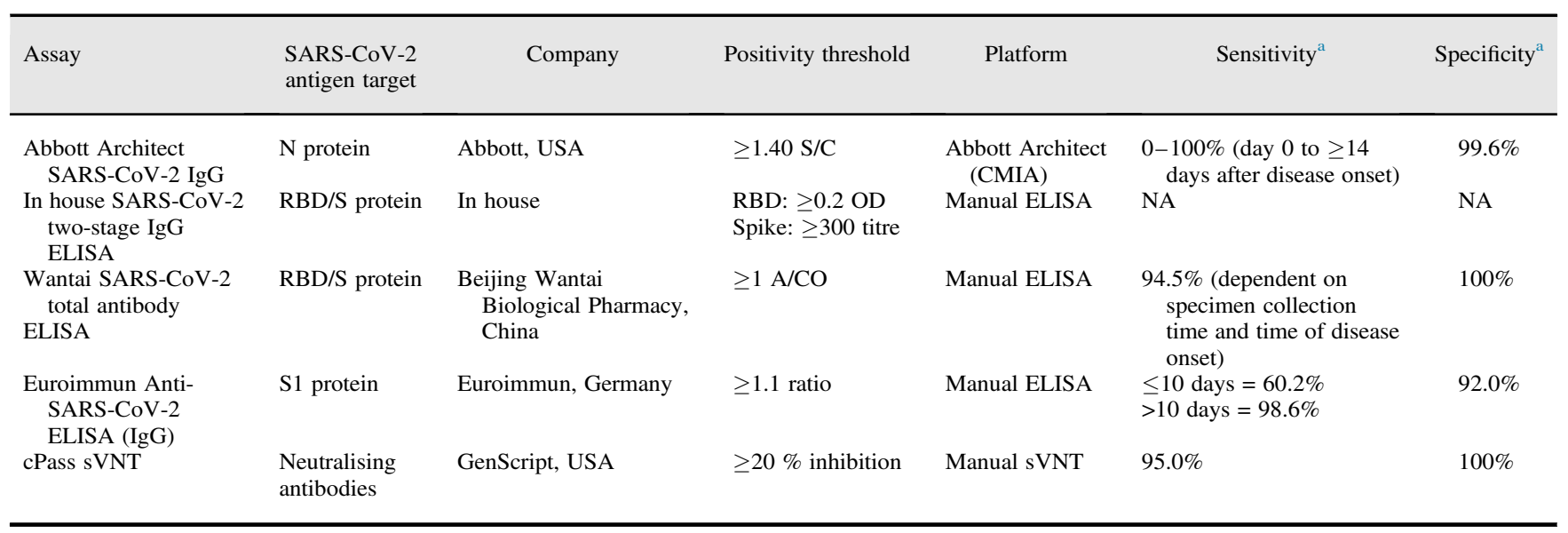

CMIA, chemiluminescent microparticle immunoassay; N, Nucleocapsid; NA, not applicable; RBD, receptor binding domain; S, Spike; sVNT, surrogate virus neutralisation test.

${ }^{a}$ Sensitivity and specificity according to manufacturer.

(95\% CI 0.0-2.0\%). Using the Abbott assay with a threshold of $0.5 \mathrm{~S} / \mathrm{C}$, the estimated prevalence is $2.0 \%(95 \% \mathrm{CI}$ $0.8-3.2 \%$ ). To incorporate the effect of secondary orthogonal testing (testing with a second assay, using a different target), we carried out a Bayesian statistical analysis for prevalence estimation. ${ }^{17}$ Applying the secondary tests to the 11 samples that tested positive on the Abbott assay with a threshold of 1.4 $\mathrm{S} / \mathrm{C}$, the estimated prevalence in the higher-risk group is $0.9 \%$ ( $95 \%$ credible interval $0.4-1.7 \%$ ). Applying the secondary tests to the 25 samples that tested positive on the Abbott assay with a threshold of $0.5 \mathrm{~S} / \mathrm{C}$, the estimated actual prevalence is $0.8 \%$ (95\% credible interval $0.4-1.5 \%$ ).

\section{Antibody detection among probable cases}

Of the nine probable cases, one was positive on four of the five assays while another was positive on three of the five assays, suggesting likely infection (Fig. 1; Supplementary Table 4, Appendix A). The remaining seven were negative by all five assays, and the Abbott assay raw values of these ranged from $0.01-0.04 \mathrm{~S} / \mathrm{C}$, suggesting that these were unlikely to have had COVID-19 infection.

\section{DISCUSSION}

Serological testing can be useful as an epidemiological tool to estimate the overall prevalence of infection in the community, a public health tool during an outbreak to identify recent infections and inform contact tracing procedures, and to assist in clinical diagnosis. ${ }^{18}$ It is also possible, once the correlates of protection against reinfection are defined, that serological testing based on the $\mathrm{S}$ protein could be used to confirm evidence of past infection or vaccination.

Using a cohort of PCR-confirmed cases to assess sensitivity, we found suboptimal performance of the Abbott assay at 11-17 weeks post-infection with a sensitivity of $76.9 \%$, somewhat lower than the previously published data ${ }^{19-22}$ and manufacturer's claim (100\% after 14 days). Several factors likely contributed to this. Firstly, most of the cases in the SDHB region were not hospitalised, and there is some evidence that antibody levels correlate with disease severity. ${ }^{23}$ Secondly, a median of 14 weeks (range 11-17 weeks) had lapsed between symptom onset and serum collection, and $\mathrm{N}$ protein antibodies have been reported to decline relatively quickly post-infection. ${ }^{24-27}$ In contrast, the sensitivity of the plate-based assays based on the $\mathrm{S}$ protein antigens was higher (89.7-94.9\%), in keeping with the notion that antibodies against the $\mathrm{S}$ protein persist for a longer duration than those to the $\mathrm{N}$ protein. ${ }^{28}$ Beyond antibody kinetics, it is also possible that the Abbott chemiluminescent immunoassay technology contributed to the reduced sensitivity observed, as studies comparing the Elecsys AntiSARS-CoV-2 assay (Roche Diagnostics, Germany) with two commercially available $S$ protein antibody assays, demonstrated $\mathrm{N}$ protein antibodies persisting as long as $\mathrm{S}$ protein antibodies out to 83 days post-symptom onset. ${ }^{29}$

Two of the PCR-confirmed cases were negative on all five serological assays. A small proportion of PCR-positive patients will be persistently negative by serology. ${ }^{30}$

In our hands, the Abbott assay specificity was $99.7 \%$ (95\% CI 98.2-99.99\%), comparable to the manufacturer's claim $(99.6 \%)$. However, given the very low prevalence of COVID-19 infection in NZ, the positive predictive value will be relatively low. Thus, we suggest an orthogonal testing algorithm as a supplemental assay before reporting results as true positives.

$\mathrm{S}$ protein is the main target for SARS-CoV-2 NAbs, which are antibodies that typically block entry of the virus into cells. ${ }^{24}$ In this study NAbs were measured using a sVNT $^{15}$ with $88.5 \%$ of the PCR-confirmed cases having detectable NAbs 11-17 weeks post-infection, with lower NAbs among those with mild symptoms. A decline in NAb levels has been noted in some recent reports, ${ }^{23}$ but further studies are needed to fully understand these immunokinetics and the implications this may have for protection against reinfection and vaccines based on the $S$ protein and RBD.

This study of over 1000 individuals who self-identified as being higher risk than the overall population for COVID-19 in our region, identified a further nine infections. Of these, 
Table 3 Sensitivity and specificity of the investigated SARS-CoV-2 assays

\begin{tabular}{|c|c|c|c|}
\hline Assay & SARS-CoV-2 antigen & Sensitivity (\%) & Specificity $(\%)$ \\
\hline Abbott Architect SARS-CoV-2 IgG (using manufacturer cut-off of $\geq 1.40$ ) & $\mathrm{N}$ protein & $\begin{array}{l}76.9(60 / 78) \\
(95 \% \text { CI } 66.0-85.7)\end{array}$ & $\begin{array}{l}99.7(299 / 300) \\
(95 \% \text { CI } 98.2-99.99)\end{array}$ \\
\hline Abbott Architect SARS-CoV-2 IgG (using revised cut-off of $\geq 0.50$ ) & $\mathrm{N}$ protein & $\begin{array}{l}94.9(74 / 78) \\
(95 \% \text { CI } 87.4-98.6)\end{array}$ & $\begin{array}{l}98.3(295 / 300) \\
(95 \% \text { CI } 96.2-99.5)\end{array}$ \\
\hline In house SARS-CoV-2 two-stage IgG ELISA & $\mathrm{RBD} / \mathrm{S}$ protein & $\begin{array}{l}91.0(71 / 78) \\
(95 \% \text { CI } 82.4-96.3)\end{array}$ & $\begin{array}{l}100(300 / 300) \\
(95 \% \text { CI } 98.8-100.0)\end{array}$ \\
\hline Euroimmun Anti-SARS-CoV-2 ELISA (IgG) ${ }^{\mathrm{a}}$ & S1 protein & $\begin{array}{l}89.7(70 / 78) \\
(95 \% \text { CI } 80.8-95.5)\end{array}$ & $\begin{array}{l}100(300 / 300) \\
(95 \% \text { CI } 98.8-100.0)\end{array}$ \\
\hline cPass sVNT & Neutralising antibodies & $\begin{array}{l}88.5 \%(69 / 78) \\
(95 \% \text { CI } 79.2-94.6)\end{array}$ & $\begin{array}{l}100 \%(300 / 300) \\
(95 \% \text { CI } 98.8-100.0)\end{array}$ \\
\hline
\end{tabular}

CI, confidence interval; N, Nucleocapsid; RBD, receptor binding domain; S, Spike; sVNT: surrogate virus neutralisation test.

${ }^{a}$ Equivocal results considered negative.

all had epidemiological risks including travel to Europe during their outbreak, and/or being a close contact of a known case. Only two of these individuals had PCR testing performed; the remaining seven did not as they were symptomatic overseas or did not meet the original case definition. Undiagnosed infection was not detected among front line healthcare workers, tourism workers, and casual contacts of known cases. It is possible that cases of infection may have been missed as the Abbott assay, which was used as our initial screening assay, demonstrated sub-optimal sensitivity. However, the grey-zone approach utilised, based on a ROC analysis, improved the sensitivity of the assay to $94.9 \%$. Given the imperfect sensitivity, and unknown prevalence among the tested population in our region, it is difficult to estimate the true number of cases of infection that may have been missed. By applying Rogan-Gladen and Bayesian estimations for actual prevalence in the higher risk group we were able to obtain more precise estimates of actual prevalence and found that the estimates do not appear to depend strongly on the threshold used in primary test, as evidenced by the concordance of the obtained estimates.

The epidemiology of COVID-19 in NZ in early 2020 is relatively unique: $\mathrm{NZ}$ is an island nation with low population density by world standards; the pandemic was well signalled overseas allowing border measures to be put in place; the pandemic coincided with the Southern Hemisphere autumn (fall); our hospitals were not overwhelmed with cases; and our setting has a well-coordinated network of microbiology laboratories and Public Health units. Therefore, it is plausible that the majority of cases were identified by targeting PCR

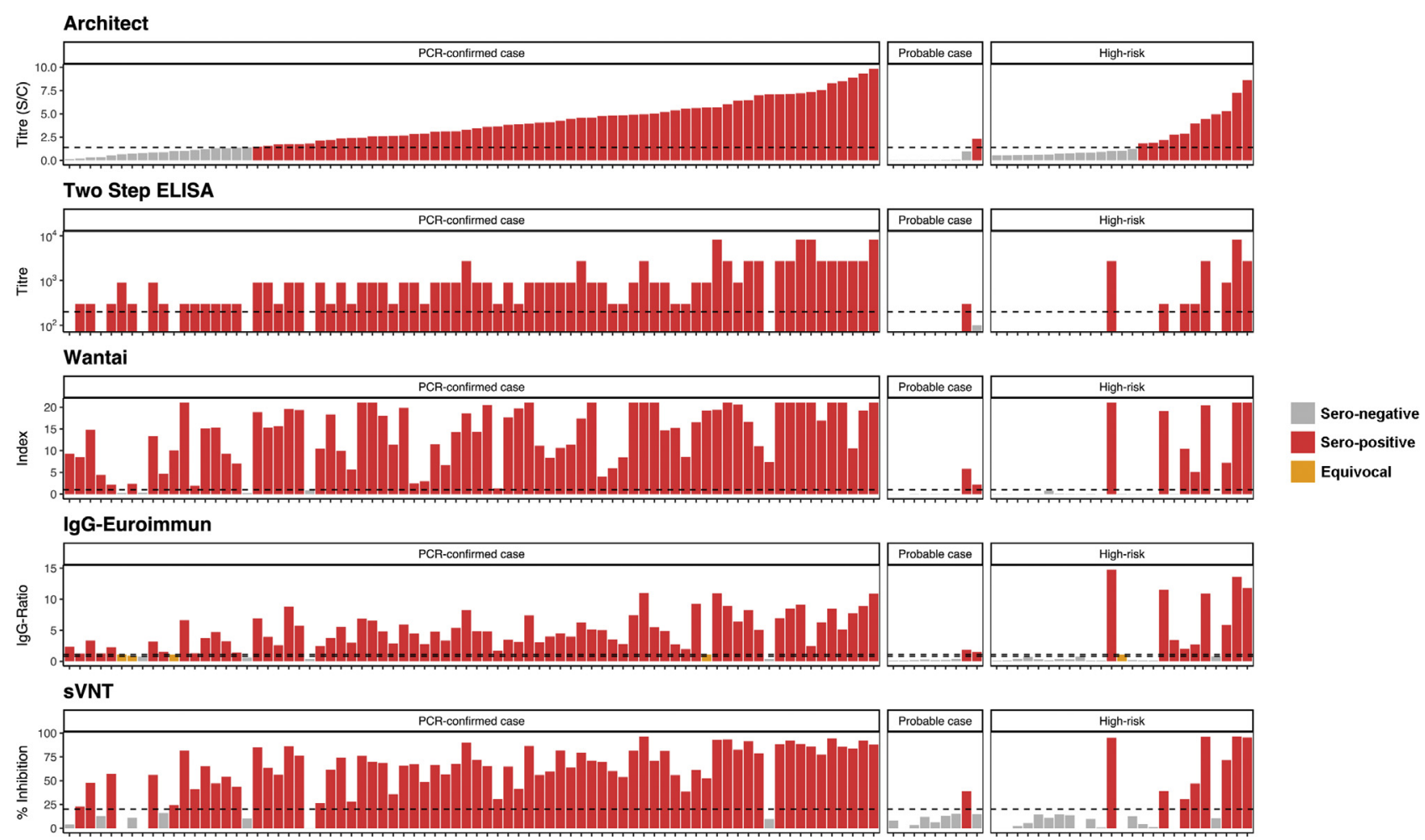

Fig. 1 Antibody levels for the examined assays for the samples tested on all five assays [all PCR-confirmed cases, all probable cases, and higher risk samples in the 'grey-zone' $(0.5-1.39 \mathrm{~S} / \mathrm{C})$ or positive $(\geq 1.4 \mathrm{~S} / \mathrm{C})$ results on the Abbott assay] ( $n=112)$. Dashed horizontal lines show assay specific cut-off. 
testing on the basis of symptoms and epidemiological risks, and were comprehensively isolated by Public Health intervention, limiting community spread.

An unexpected finding was that seven of the nine individuals diagnosed with 'probable' infection, and included in NZ's official tally, were sero-negative despite being tested on all five assays. While acknowledging the delay (approximately 3 months) in serum collection and the possible impact on sensitivity, it is likely that at least some of these individuals did not have infection. This highlights the role of serology in the diagnostic algorithm where PCR is negative despite symptoms and epidemiological risks, and further testing of NZ's remaining 341 probable cases may be warranted.

We note the high female predominance in this study $(75 \%)$. We hypothesise this is due to the high proportion of frontline healthcare workers $(62 \%)$, which is a female dominated occupation, and it is possible females are more willing to participate in such studies.

Our study has some limitations. Firstly, the antenatal sera used to determine specificity is not representative of the general population. Secondly, the delay in specimen collection after the outbreak likely had an impact on the Abbott assay sensitivity. We cannot be certain that undiagnosed cases were not missed using the Abbott assay as our screening test. However, every effort was made to mitigate against this by lowering the cut-off for the initial Abbott screening assay. Lastly, it is important to note that this is not a sero-prevalence study. Participants who selfidentified as higher risk were actively recruited, therefore the sero-positivity rate calculated in this group in the SDHB region cannot be extrapolated to the general population.

\section{CONCLUSION}

In conclusion, our study shows that the COVID-19 outbreak in the SDHB region in early 2020, despite the testing restrictions early on in the outbreak, was largely confined to the PCR-confirmed cases and those identified as at higher risk due to recent travel and/or close contact with a known case. We found little evidence of undetected infection among the individuals in the SDHB region who were considered to be at higher risk than the average resident, due to contact with a PCR-confirmed case, or because of workplace duties, or because they were Queenstown residents. The $\mathrm{N}$ proteinbased Abbott assay demonstrated the lowest sensitivity of the assays investigated, likely impacted by the delay in serum collection, which appears to affect $\mathrm{N}$ protein antibodies over $\mathrm{S}$ protein antibodies. Whilst this may lead to missed cases, the utility of a high throughput system for large scale testing does, to a degree, offset this significant limitation, especially when combined with secondary $\mathrm{S}$ protein assays of higher sensitivity. When designing a SARS-CoV-2 serological assay algorithm, the purpose of testing is a major consideration, with different assay combinations suitable for highthroughput sero-prevalence purposes versus individual level clinical diagnostics.

Acknowledgements: We thank the team behind the Southern COVID-19 Serology Study for facilitating sample collection, delivery, and processing for the SARS-CoV-2 serological testing. We thank Linfa Wang at Duke-NUS and GenScript for providing sVNT testing kits and technical advice. We are grateful to Drs Campbell Sheen (Callaghan Innovation) and James Dickson (University of Auckland) for providing antigens for the in house ELISA.

Conflicts of interest and sources of funding: Sample collection, processing, and Abbott Architect consumables were funded by Southern Community Laboratories. This work was also funded in part by the School of Medicine Foundation (University of Auckland) and the COVID-19 Innovation Acceleration Fund (Ministry of Business, Innovation and Employment). The authors state that there are no conflicts of interest to disclose.

\section{APPENDIX A. SUPPLEMENTARY DATA}

Supplementary data to this article can be found online at https://doi.org/10.1016/j.pathol.2021.04.001.

Address for correspondence: Dr Arlo Upton, Southern Community Laboratories, PO Box 6064, Dunedin, 9059, New Zealand. E-mail: arlo.upton@ sclabs.co.nz

\section{References}

1. Zhu N, Zhang D, Wang W, et al. A novel coronavirus from patients with pneumonia in China, 2019. N Engl J Med 2020; 382: 727-33.

2. World Health Organization. Timeline: WHO's COVID-19 response. Cited 4 Sep 2020. https://www.who.int/emergencies/diseases/novelcoronavirus-2019/interactive-timeline/\#!

3. John Hopkins University. Coronavirus resource centre. Cited 5 Aug 2020. https://coronavirus.jhu.edu/

4. New Zealand Ministry of Health. COVID-19 - current cases. Cited 5 Aug 2020. https://www.health.govt.nz/our-work/diseases-andconditions/covid-19-novel-coronavirus/covid-19-current-situation/ covid-19-current-cases

5. Strongman S. COVID-19 pandemic timeline. Cited 5 Aug 2020. https:// shorthand.radionz.co.nz/coronavirus-timeline/index.html

6. New Zealand Government. COVID-19 alert system. Cited 16 Jan 2021 https://covid19. govt.nz/alert-system/

7. Tang YW, Schmitz JE, Persing DH, et al. Laboratory diagnosis of COVID-19: current issues and challenges. J Clin Microbiol 2020; 58: e00512-20.

8. Jiang H-W, Li Y, Zhang H-N, et al. SARS-CoV-2 proteome microarray for global profiling of COVID-19 specific IgG and IgM responses. Nat Commun 2020; 11: 3581.

9. Jefferies S, French N, Gilkison C, et al. COVID-19 in New Zealand and the impact of the national response: a descriptive epidemiological study. Lancet Public Health 2020; 5: e612-23.

10. New Zealand Ministry of Health. Case definition and testing guidance for COVID-19. Cited 16 Jan 2021. https://www.health.govt.nz/ourwork/diseases-and-conditions/covid-19-novel-coronavirus/covid-19information-health-professionals/case-definition-and-testing-guidancecovid-19

11. Harris PA, Taylor R, Thielke R, et al. Research electronic data capture (REDCap)-a metadata-driven methodology and workflow process for providing translational research informatics support. J Biomed Inform 2009; 42: 377-81.

12. Corman VM, Landt O, Kaiser M, et al. Detection of 2019 novel coronavirus (2019-nCoV) by real-time RT-PCR. Euro Surveill 2021; 26: 210204e.

13. Stadlbauer D, Amanat F, Chromikova V, et al. SARS-CoV-2 seroconversion in humans: a detailed protocol for a serological assay, antigen production, and test setup. Curr Protoc Microbiol 2020; 57: e100.

14. McGregor R, Whitcombe A, Sheen C, et al. Collaborative networks enable the rapid establishment of serological assays for SARS-CoV2 during nationwide lockdown in New Zealand. PeerJ 2020; 8: e9863.

15. Tan CW, Chia WN, Qin X, et al. A SARS-CoV-2 surrogate virus neutralization test based on antibody-mediated blockage of ACE2-spike protein-protein interaction. Nat Biotechnol 2020; 38: 1073-8.

16. Rogan WJ, Gladen B. Estimating prevalence from the results of a screening test. Am J Epidemiol 1978; 107: 71-6.

17. Flor M, Weiß M, Selhorst $T$, et al. Comparison of Bayesian and frequentist methods for prevalence estimation under misclassification. BMC Public Health 2020; 20: 1135. 
18. Yong SEF, Anderson DE, Wei WE, et al. Connecting clusters of COVID-19: an epidemiological and serological investigation. Lancet Infect Dis 2020; 20: 809-15.

19. Bryan A, Pepper G, Wener MH, et al. Performance characteristics of the Abbott Architect SARS-CoV-2 IgG assay and seroprevalence in Boise, Idaho. J Clin Microbiol 2020; 58:e00941-20.

20. Chew KL, Tan SS, Saw S, et al. Clinical evaluation of serological IgC antibody response on the Abbott Architect for established SARS-CoV-2 infection. Clin Microbiol Infect 2020; 26: 1256.e9-e11.

21. Public Health England. Evaluation of the Abbott SARS-CoV-2 IgG for the detection of anti-SARS-CoV-2 antibodies. Cited 4 Sep 2020. https:// assets.publishing.service.gov.uk/government/uploads/system/uploads/ attachment data/file/890566/Evaluation_of_Abbott_SARS_CoV_2 IgG_PHE.pdf

22. The National SARS-CoV-2 Serology Assay Evaluation Group. Performance characteristics of five immunoassays for SARS-CoV-2: head-to-head benchmark comparison. Lancet Infect Dis 2020; 20: $1390-400$.

23. Seow J, Graham C, Merrick B, et al. Longitudinal observation and decline of neutralizing antibody responses in the three months following SARS-CoV-2 infection in humans. Nat Microbiol 2020; 5 : $1598-607$.
24. Grandjean L, Saso A, Ortiz A, et al. Humoral response dynamics following infection with SARS-CoV-2. medRxiv 2020; $22 \mathrm{Jul}$ : https:// doi.org/10.1101/2020.07.16.20155663.

25. Whitcombe A, McGregor R, Craigie A, et al. Comprehensive analysis of SARS-CoV-2 antibody dynamics in New Zealand. MedRxiv 2020; 11 Dec: https://doi.org/10.1101/2020.12.10.20246751.

26. Ripperger TJ, Uhrlaub JL, Watanabe M, et al. Orthogonal SARS-CoV-2 serological assays enable surveillance of low-prevalence communitie and reveal durable humoral immunity. Immunity 2020; 53.925-33e4.

27. Henss L, Scholz T, von Rhein C, et al. Analysis of humoral immune responses in patients with severe acute respiratory syndrome coronavirus 2 infection. J Infect Dis 2021; 223: 56-61.

28. Wajnberg A, Amanat F, Firpo A, et al. SARS-CoV-2 infection induces robust, neutralizing antibody responses that are stable for at least three months. medRxiv 2020; 17 Jul: https://doi.org/10.1101/ 2020.07.14.20151126.

29. Chua KYL, Vogrin S, Bittar I, et al. Clinical evaluation of four com mercial immunoassays for the detection of antibodies against establishec SARS-CoV-2 infection. Pathology 2020; 52: 778-82.

30. Oved K, Olmer L, Shemer-Avni Y, et al. Multi-center nationwide comparison of seven serology assays reveals a SARS-CoV-2 non-responding seronegative subpopulation. EClinicalMedicine 2020; 29: 100651. 